\title{
DELAYED VERSUS ACCELERATED WEIGHT-BEARING REHABILITATION PROTOCOL FOLLOWING ANTERIOR CRUCIATE LIGAMENT RECONSTRUCTION: A SYSTEMATIC REVIEW AND META-ANALYSIS
}

Zheyuan FAN, MD, Jingtong YAN, MD, Zhongsheng ZHOU, MD, Yu GAO, MD, Jinshuo TANG, MD, Yuhuan LI, MD, Zhuo ZHANG, MD, Modi YANG, MD and Jiayin LV MD

From the Department of Orthopedics, China-Japan Union Hospital of Jilin University, Changchun, Jilin, China

Objective: To compare accelerated and delayed weight-bearing rehabilitation of anterior cruciate ligament reconstruction regarding clinical outcome measures of knee function (International Knee Documentation Committee Subjective Knee Form (IKDC), Lysholm Knee Scoring Scale, Tegner Activity Scale, and Knee Injury and Osteoarthritis Outcome Score (KOOS)), knee laxity, range of movement, quadriceps, and bone tunnel enlargement.

Design: Systematic review and meta-analysis.

Methods: Systematic searches of Embase, MEDLINE, CINAHL, and the Cochrane Library databases, from inception to February 2021, for studies comparing delayed or accelerated weight-bearing rehabilitation protocol after anterior cruciate ligament reconstruction in adult patients.

Results: Nine studies met the eligibility criteria. A meta-analysis revealed a higher risk of knee laxity in the accelerated weight-bearing group. Accelerated weight-bearing may be related to higher IKDC scores, while there was no statistical difference for Lysholm, Tegner, and KOOS scores at a follow-up within $\mathbf{2}$ years. Four of $\mathbf{5}$ studies reported no statistical difference for quadriceps strength and range of movement scores, while 2 studies reported bone tunnel enlargement in the accelerated weight-bearing group.

Conclusion: This systematic review confirmed that accelerated weight-bearing caused more serious knee laxity and bone tunnel widening than delayed weight-bearing after anterior cruciate ligament reconstruction. We therefore recommend that clinicians should select postoperative rehabilitation programmes with caution.

Key words: anterior cruciate ligament reconstruction; rehabilitation; weight-bearing; knee laxity.

PROSPERO registration number: CRD42021249521. Accepted Dec 21, 2021; Epub ahead of print Jan 17, 2021

J Rehabil Med 2022; 54: jrm00260

Correspondence address: Modi Yang and Jiayin Lv, Department of Orthopedics, China-Japan Union Hospital of Jilin University, 126 Xiantai Street, Changchun 130033, P. R. China. E-mail: yangmodi@jlu.edu.cn and Ivjy@jlu.edu.cn

$\mathrm{A}$ nterior cruciate ligament (ACL) rupture is a serious condition. ACL reconstruction (ACLR) is becoming the most common therapy for ACL-deficient individuals following advances in basic research, sur-

\section{LAY ABSTRACT}

Anterior cruciate ligament rupture is one of the most common sports injuries. This systematic review confirmed that accelerated weight-bearing rehabilitation resulted in more serious knee laxity and bone tunnel widening than delayed weight-bearing rehabilitation after ACLR. We therefore recommend that clinicians should select postoperative rehabilitation programmes with caution.

gical devices, and surgical techniques (1). We recommend ACLR for patients who have other combined injuries, functional instability of the knee joint, a completely broken ACL, or avulsion fractures. Reconstruction is beneficial in restoring knee stability and reducing the risk of subsequent chondral or meniscal damage in the long term. The lack of consensus on the optimal rehabilitation protocol and weight-bearing after ACL reconstruction remains of interest for further research (2). Reports range from immediate weightbearing to delayed weight-bearing for up to 8 weeks.

Weight-bearing training facilitates isometric activity of the muscles surrounding the knee joint, reduces knee joint effusions, increases earlier articular cartilage compression and nutrition, maintains subchondral bone strength, and decreases peripatellar fibrosis (3-7). Proprioception and joint awareness may continue to weaken after ACL injuries or reconstruction. Simulated loading behaviours do not match loading abilities after ACLR. The ACLR limb may experience lower peak patellofemoral joint loading during running because of an anterior shift in the foot ground centre of pressure until 24 months (6). It might seem appropriate to resume weight-bearing exercises and normal gait as soon as possible. However, because of the inherent tilt of the tibial plateau, excessive compressive loads caused by impact loads along the tibial shaft may lead to tears of the ACL when the knee is weight-bearing, and the risk of loss of graft fixation and subsequent instability may increase (8). An accelerated rehabilitation protocol can also increase bone tunnel enlargement after ACLR using hamstrings autograft, because of the bungee cord effect associated with longitudinal graft motion and the windshield wiper motion effect associated with transverse graft motion $(1,3)$.

The aim of this study was to systematically review the literature and compare measures of knee function 
(including IKDC, KOOS, Tegner, and Lysholm scores), knee laxity, range of motion (ROM), quadriceps, and bone tunnel enlargement between delayed and accelerated weight-bearing rehabilitation protocols.

\section{METHODS}

\section{Protocol and registration}

The study was performed according to the Preferred Reporting Items for Systematic Review and Meta-Analysis (PRISMA) guidelines (9). The protocol was registered in the International Prospective Register of Systematic Reviews (PROSPERO) and met all the eligibility criteria for protocol registration (registration number: CRD42021249521).

\section{Eligibility criteria}

The PICO (P: patient, I: interventions, C: comparisons, O: outcomes) was defined as primary ACLR in adults treated with a delayed weight-bearing or accelerated weight-bearing rehabilitation protocol with a clinical evaluation index. The inclusion criteria were studies that: included a comparison of delayed or accelerated rehabilitation protocols in patients after ACLR; restricted the operative method to arthroscopic ACLR; included a patient mean age of $\geq 18$ years; were randomized, controlled, retrospective, or prospective trials. Exclusion criteria were: studies that were not published in English language; studies with a follow-up time of less than 3 months; and studies of patients with multiple ligament injuries.

\section{Information sources and literature search}

A literature search was performed in February 2021 using 4 electronic databases: Embase, MEDLINE, Web of Science, and the Cochrane Library. The search strategy included the key term "anterior cruciate ligament reconstruction" in combination with delayed and accelerated rehabilitation protocol search terms and outcomes. A sensitive search strategy was used in order to include all relevant literature, as follows:

- Topics (TS) = (anterior cruciate ligament)

- TS = (surgery OR operation OR reconstruction OR transplantation)

- $\mathrm{TS}=$ (weight-bearing $\mathrm{OR}$ rehabilitation $\mathrm{OR}$ weight training)

- TS= (time OR timing OR delay OR early OR accelerate)

\section{Study selection and data extraction}

Search results were extracted to EndNote X9 software (Clarivate Analytics, Philadelphia, Pennsylvania, USA).

Outcomes included IKDC, KOOS, Lysholm, Tegner scores, ROM of knee joint, anteroposterior (A-P) laxity measured by KT-1000 arthrometer, quadriceps strength and bone tunnel enlargement. Outcomes were classified according to relevance to clinical decision-making. The primary reviewer extracted all relevant data, and a second reviewer checked for accuracy as part of the quality control.

\section{Methodological and quality assessment}

The risk of bias of each study was assessed by the criteria outlined in the Cochrane Handbook for Systematic Reviews of Interventions (Higgins 2011) (10).

\section{Data analysis}

Review Manager (V.5.4, The Cochrane Collaboration, London, UK) was used for analysis of the selected studies. The mean and standard deviation of scores about the clinical outcome and A-P laxity measured by KT-1000 were extracted from the studies and provided in the form of a forest plot. Statistical significance was defined as $p \leq 0.05$. Estimation of the heterogeneity of pooled data was performed using $\chi^{2}$ and $\mathrm{I}^{2}$ tests. When the $\mathrm{I}^{2}$ test outcome was less than $50 \%$, a fixed effects meta-analysis was chosen.

\section{RESULTS}

\section{Study selection}

In total, 911 studies were eligible (Fig. 1), but only 9 studies qualified for inclusion $(1,8,11-17)$ (Table I). Due to the considerable variation in rehabilitation protocols across studies, for the purposes of the current study accelerated weight-bearing was defined as full weight-bearing within 2 weeks, and delayed weightbearing was defined as full weight-bearing beyond 2 weeks. The main reason for excluding studies was that the timing of postoperative weight-bearing training was not an independent variable. Studies of multiple ligament injuries were excluded. Also excluded was 1 study that compared accelerated and delayed rehabilitation, showing the total duration of rehabilitation, but without a coherent plan regarding weight-bearing (18).

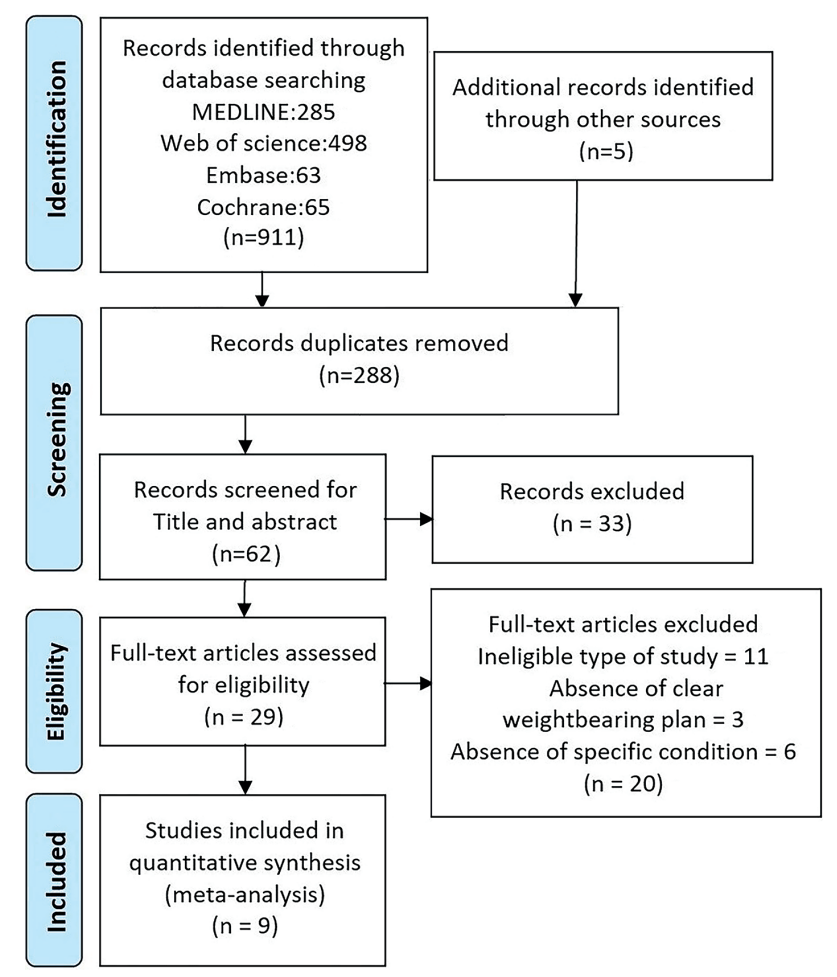

Fig. 1. Preferred Reporting Items for Systematic Review and MetaAnalysis (PRISMA) flow diagram of the study screening and selection. 
Table I. Study and patient characteristics

\begin{tabular}{|c|c|c|c|c|c|c|c|c|c|c|c|c|}
\hline \multirow[b]{2}{*}{ Study } & \multirow[b]{2}{*}{ Study design } & \multirow{2}{*}{$\begin{array}{l}\text { Follow-up } \\
\text { (months) }\end{array}$} & \multicolumn{2}{|c|}{$\begin{array}{l}\text { Time, injury to surgery } \\
\text { (days) }\end{array}$} & \multicolumn{2}{|l|}{$\underline{n}$} & \multicolumn{2}{|l|}{ Age (years) } & \multicolumn{2}{|c|}{ BMI $\left(\mathrm{kg} / \mathrm{m}^{2}\right)$} & \multicolumn{2}{|c|}{$\begin{array}{l}\operatorname{Sex}(m, \\
n) /(f, n)\end{array}$} \\
\hline & & & $\mathrm{D}$ & A & $\mathrm{D}$ & A & $\mathrm{D}$ & A & D & A & $\mathrm{D}$ & A \\
\hline $\begin{array}{l}\text { Di Miceli, et al. } 2017 \\
\text { (11) }\end{array}$ & Retrospective & $42 \pm 21.6$ & $\mathrm{~N} / \mathrm{A}$ & $\mathrm{N} / \mathrm{A}$ & 6 & 16 & $31.4 \pm 10.8$ & $30.2 \pm 10.5$ & N/A & N/A & $\mathrm{N} / \mathrm{A}$ & $\mathrm{N} / \mathrm{A}$ \\
\hline Zhu, et al. 2012 (13) & Retrospective & $3,6,12$ & N/A & N/A & 15 & 15 & N/A & N/A & N/A & N/A & $\mathrm{N} / \mathrm{A}$ & N/A \\
\hline $\begin{array}{l}\text { Beynnon, et al. } 2005 \\
\text { (16) }\end{array}$ & RCT & $3,6,12,24$ & $124(48-338)$ & $90.7(36-260)$ & 14 & 11 & $34.7(19-44)$ & $30.4(18-44)$ & $\mathrm{N} / \mathrm{A}$ & N/A & $6 / 6$ & $5 / 5$ \\
\hline $\begin{array}{l}\text { Henriksson, et al. } \\
2002 \text { (17) }\end{array}$ & Prospective & $6,12,24$ & $270(15-1,320)$ & $165(30-1,950)$ & 23 & 22 & $24 \pm 6$ & $24 \pm 5$ & N/A & N/A & $7 / 16$ & $18 / 4$ \\
\hline Tajima, et al. 2019 (1) & Prospective & 12 & N/A & N/A & 18 & 19 & $23.3(15-48)$ & $23.9(15-40)$ & N/A & N/A & $7 / 11$ & $9 / 10$ \\
\hline Tyler, et al. 1998 (8) & RCT & $7.3(6-14)$ & N/A & N/A & 20 & 25 & N/A & N/A & N/A & N/A & $\mathrm{N} / \mathrm{A}$ & N/A \\
\hline $\begin{array}{l}\text { Beynnon, et al. } 2011 \\
\text { (15) }\end{array}$ & RCT & $3,6,12,24$ & $66 \pm 31.6$ & $56 \pm 23.5$ & 17 & 19 & $30.2 \pm 9.9$ & $29.7 \pm 10.1$ & N/A & N/A & $9 / 8$ & $13 / 6$ \\
\hline
\end{tabular}

(15)

BMI: body mass index; f: female; m: male; N/A: not available; D: delayed group; A: accelerated group; RCT: randomized controlled trial.

\section{Risk of bias and quality assessment}

Most of the included studies exhibited a moderate risk of bias, but were of acceptable quality. The overall risk of bias and quality of the studies are shown in Fig. 2. Seven studies were assessed as low risk (77.8\%) and 2 as high risk (22.2\%) in random sequence generation. Six studies were assessed as low risk (66.7\%), and 1 as high risk $(11.1 \%)$ in allocation concealment. Three studies were assessed as low risk (33.3\%) and 5 as unclear risk $(55.6 \%)$ in blinding of participants and personnel. Five studies were assessed as low risk (55.6\%) and 2 as high risk $(22.2 \%)$ in outcome assessment blinding. Six studies were assessed as low risk $(66.7 \%)$ and 3 as unclear risk $(33.3 \%)$ in incomplete outcome data. Seven studies were assessed as low risk (77.8\%) and 1 as high risk (11.1\%) in selective reporting. As for other biases, 5 studies were assessed as low risk (55.6\%) and 4 as unclear risk (44.4\%).

\section{Clinical outcome measures}

The 9 studies reported IKDC scores (11-14), with 2 studies excluded in the meta-analysis $(15,16)$ (mean and SD were not provided). One study reported that the accelerated weight-bearing group showed a better score (12). The current meta-analysis revealed that early accelerated and aggressive weight-bearing after ACLR (Fig. 3) improved IDKC scores (IDKC score $p=0.001)$. Three studies reported Lysholm scores, and 1 study (8) concluded that accelerated weight-bearing may elevate Lysholm scores (Fig. 4). Meta-analysis showed no statistical difference between the 2 groups. There was no statistical difference between Tegner (1, 8, 15, 16) (Fig. 5) and $\operatorname{KOOS~}(15,16)$ (Fig. 6).

\section{Knee laxity}

A-P knee laxity (difference compared with the contralateral limb) was measured by a KT-1000 arthrometer in 7 studies $(1,8,12,14-17)$ (Fig. 7). Two of the 7 studies reported that accelerated weight-bearing may cause a greater degree of knee laxity $(1,16)$. One study reported that A-P displacement, the coupled internalexternal and varus-valgus rotations of the tibia relative to the femur all occur in varying degrees of relaxation (15). Meta-analysis of 106 cases of delayed weightbearing and 116 cases of accelerated weight-bearing

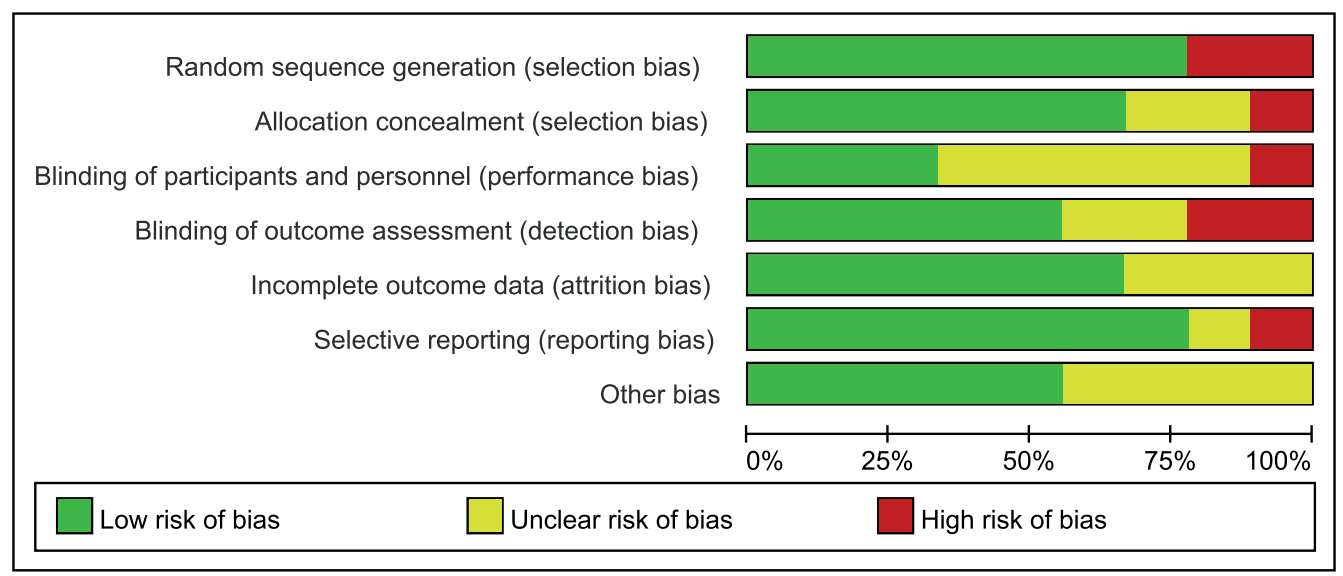

Fig. 2. Risk of bias graph. 


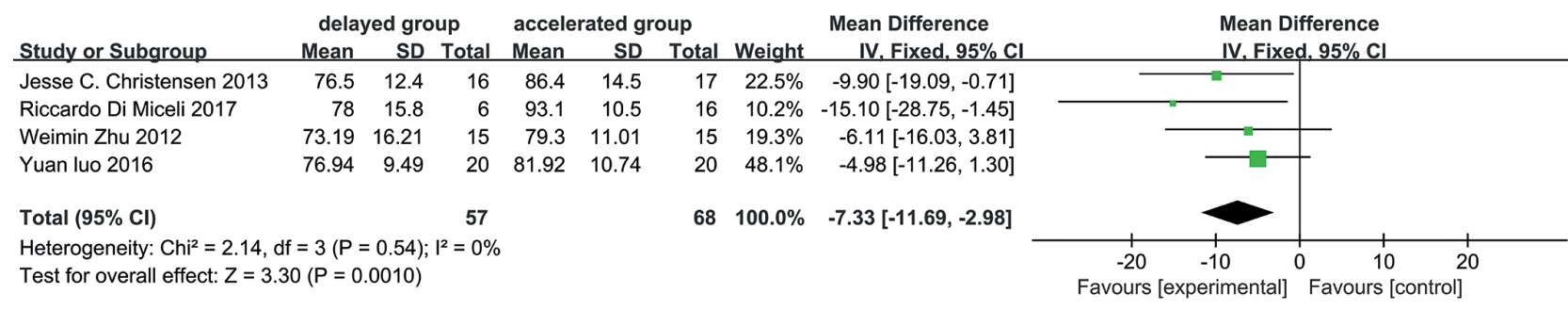

Fig. 3. Pooled analysis for International Knee Documentation Committee Subjective Knee Form (IKDC) scores. SD: standard deviation; $95 \%$ CI: 95\% confidence interval; IV: inverse variance method.

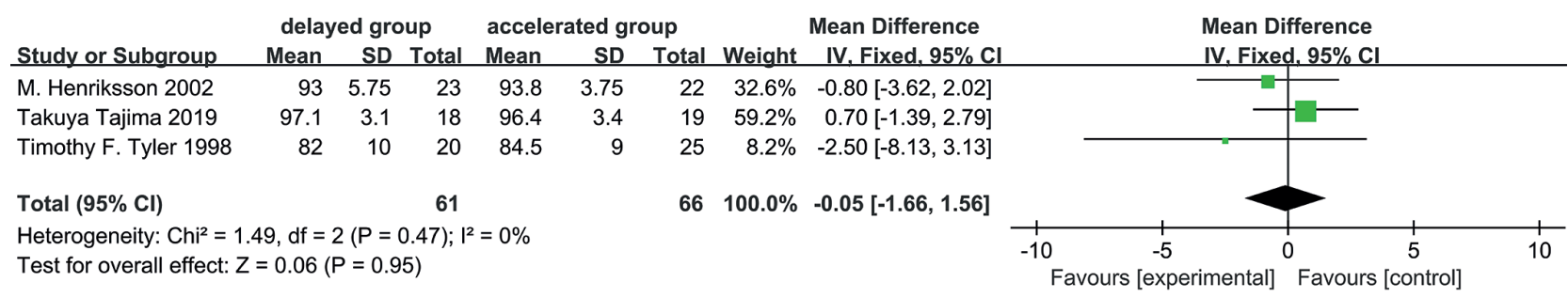

Fig. 4. Pooled analysis for Lysholm scores. SD: standard deviation; $95 \% \mathrm{CI}$ : $95 \%$ confidence interval; IV: inverse variance method.

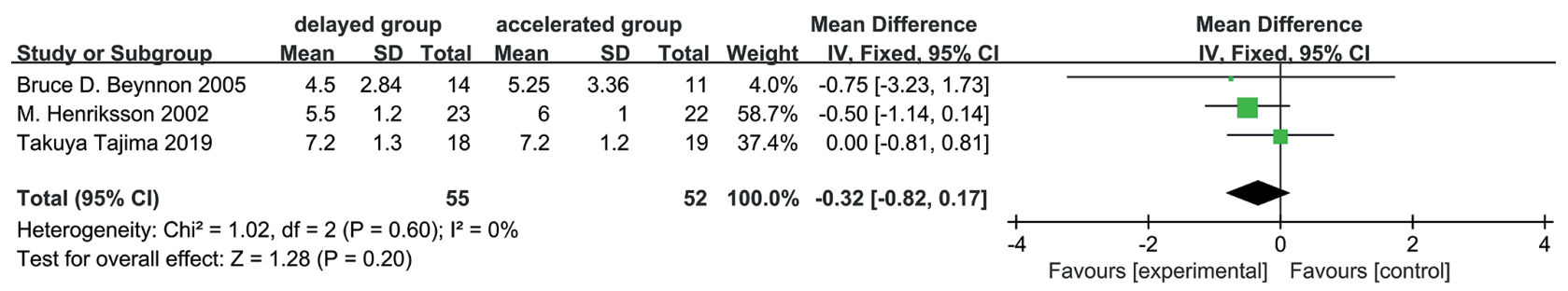

Fig. 5. Pooled analysis for Tegner scores. SD: standard deviation; 95\% CI: 95\% confidence interval; IV: inverse variance method.

showed that more significant knee laxity correlated with accelerated weight-bearing.

\section{Range of motion}

Five studies reported ROM of the knee $(1,8,13,15$, 17). Three studies found that the 2 groups showed a similar outcome for ROM $(1,8,15)$. One study found that the accelerated weight-bearing group had a better performance in the first 5 months, but the gap between the 2 groups gradually disappeared with follow-up (17). Only 1 study reported that the accelerated weightbearing group had a better performance of ROM than the delayed weight-bearing group (13).

\section{Quadriceps strength}

Quadriceps strength was compared between the 2 groups in 5 studies $(1,13-15,17)$. One study reported that the strength of the extensors was significantly greater for patients in the accelerated weight-bearing group than in the delayed group at 3 months, but no difference was observed between the groups at 6 months and beyond (15). One study found that more obvious muscle wasting was observed at 1-year follow-up in the delayed weight-bearing group (13). Other studies showed no statistical significance $(1,14,17)$.

\section{Bone tunnel enlargement}

Two studies reported data on bone tunnel enlargement $(1,13)$. Two studies used single- and double-bundle reconstructions, respectively, and all patients in the accelerated weight-bearing group showed bone tunnel enlargement. It should be noted that the enlargement of the tunnel was observed in the femur.

\section{Intervention description}

We performed surgical interventions within 1 day to 6 months after the initial injury. Surgical techniques were divided into 2 types, single-bundle $(8,11-17)$ and double-bundle (1), with surgery performed arthroscopically in all studies.

In 5 studies, the accelerated weight-bearing group followed ROM exercise of the knee, while the delayed weight-bearing group immobilized the knee joint in the initial postoperative period (13-17). In 1 study, the knee was immobilized with the knee positioned in $20^{\circ}$ of flexion to avoid excess stress on the grafts (1). 


\section{A}

delayed group accelerated group Mean SD Total

Study or Subgroup Bruce D. Beynnon 2005 Bruce D. Beynnon 2011

Total $(95 \% \mathrm{Cl})$

$\begin{array}{lllll}82.7 & 14.9 & 14 & 85\end{array}$
$88 \quad 13 \quad 18$

32

Test for overall effect: $Z=0.11(P=0.92)$
Mean Difference

\begin{tabular}{rrr}
\multicolumn{1}{c}{$\begin{array}{c}\text { Mean Difference } \\
\text { Total }\end{array}$} & Weight & IV. Fixed, 95\% Cl \\
11 & $43.1 \%$ & $-2.30[-14.16,9.56]$ \\
24 & $56.9 \%$ & $1.00[-9.33,11.33]$ \\
35 & $100.0 \%$ & $-0.42[-8.21,7.36]$
\end{tabular}

$35 \quad 100.0 \% \quad-0.42[-8.21,7.36]$

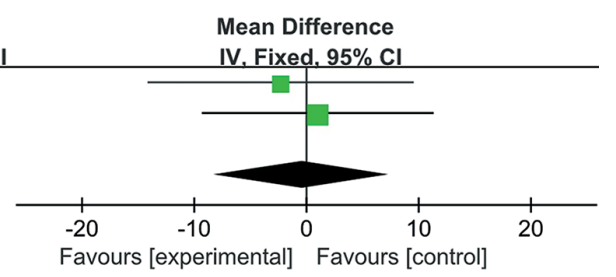

B

delayed group accelerated group Mean Difference \begin{tabular}{lllllllll} 
Bruce D. Beynnon 2005 & 76.2 & 19.5 & 14 & 73.8 & 13.2 & 11 & $40.7 \%$ & 2.40 \\
\hline
\end{tabular} $\begin{array}{lllllllll}\text { Bruce D. Beynnon } 2011 & 75 & 17 & 18 & 81 & 18 & 24 & 59.3 \% & -6.00\end{array}$

Total $(95 \% \mathrm{Cl})$

Heterogeneity: $\mathrm{Chi}^{2}=0.97, \mathrm{df}=1(\mathrm{P}=0.32) ; \mathrm{I}^{2}=0 \%$

Test for overall effect: $Z=0.62(P=0.54)$

Favours [experimental] Favours [control]

\section{C}

Study or Subgroup

Bruce D. Beynnon 2005

Bruce D. Beynnon 2011

Total $(95 \% \mathrm{Cl})$

Heterogeneity: $\mathrm{Chi}^{2}=0.00, \mathrm{df}=1(\mathrm{P}=1.00) ; \mathrm{I}^{2}=0 \%$

Test for overall effect: $Z=1.92(P=0.06)$

32 Mean SD Total Mean SD Total Weight IV. Fixed, 95\% Cl 32

$35 \quad 100.0 \% \quad-2.58[-10.78,5.62]$

delayed group accelerated group Mean Difference

Mean SD Total Mean SD Total Weight IV. Fixed. $95 \% \mathrm{Cl}$

$\begin{array}{rrrrrrrr}99.3 & 1.1 & 14 & 97.3 & 3.6 & 11 & 86.2 \% & 2.00[-0.20,4.20] \\ 98 & 4 & 18 & 96 & 13 & 24 & 13.8 \% & 2.00[-3.52,7.52]\end{array}$

$35 \quad 100.0 \% \quad 2.00[-0.05,4.05]$
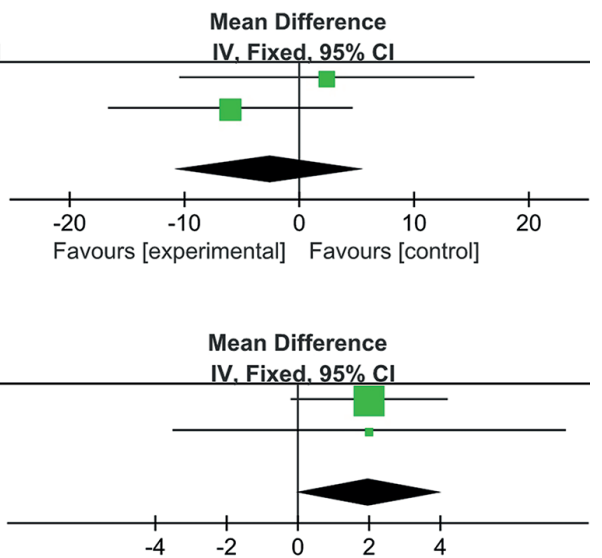

\section{D}

\section{Study or Subgroup}

Bruce D. Beynnon 2005 Bruce D. Beynnon $2011 \quad 91 \quad 10 \quad 18 \quad 92 \quad 12 \quad 24 \quad 45.3 \% \quad-1.00\left[\begin{array}{llll}-7.66,5.66 & \end{array}\right.$

delayed group accelerated group Mean SD Total Mean

Mean Difference $\begin{array}{rrrrrrrr}95.6 & 5.1 & 14 & 93.6 & 9.2 & 11 & 54.7 \% & 2.00[-4.06,8.06]\end{array}$

Total $(95 \% \mathrm{Cl})$ 32

Heterogeneity: $\mathrm{Chi}^{2}=0.43, \mathrm{df}=1(\mathrm{P}=0.51) ; \mathrm{l}^{2}=0 \%$

Test for overall effect: $Z=0.28(P=0.78)$

$35 \quad 100.0 \% \quad 0.64[-3.84,5.12]$

Favours [experimental] Favours [control]

\section{E}

Study or Subgroup delayed group accelerated group

Bruce D. Beynnon 2005 Bruce D. Beynnon 2011 Mean SD Total Mean

SD Tota

$\begin{array}{ll}\text { Mean Difference } & \text { Meal Weight } \\ \text { IV, Random, 95\% }\end{array}$

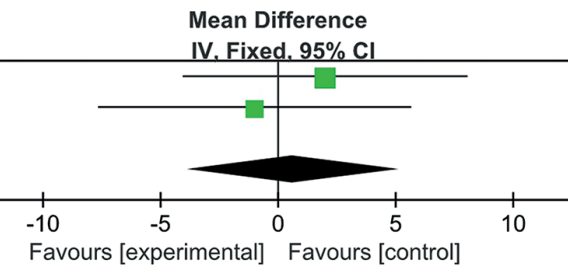

Total $(95 \% \mathrm{Cl})$ $\begin{array}{rrrr}83 & 16 & 18 & 92\end{array}$

$5.9-11 \quad 55.1 \%$

$0.50[-4.91,5.91]$

$24 \quad 44.9 \% \quad-9.00[-17.05,-0.95]$

$35 \quad 100.0 \% \quad-3.76[-13.02,5.50]$

Test for overall effect: $Z=0.80(P=0.43)$

32 $3 \%$

Fig. 6. Pooled analysis for Knee Injury and Osteoarthritis Outcome Score (KOOS) scores analyses. (A) Sports and recreation; (B) Quality of life; (C) Activities of daily living analyses; (D) Pain; (E) Symptoms; SD: standard deviation; 95\% CI: 95\% confidence interval; IV: inverse variance method.

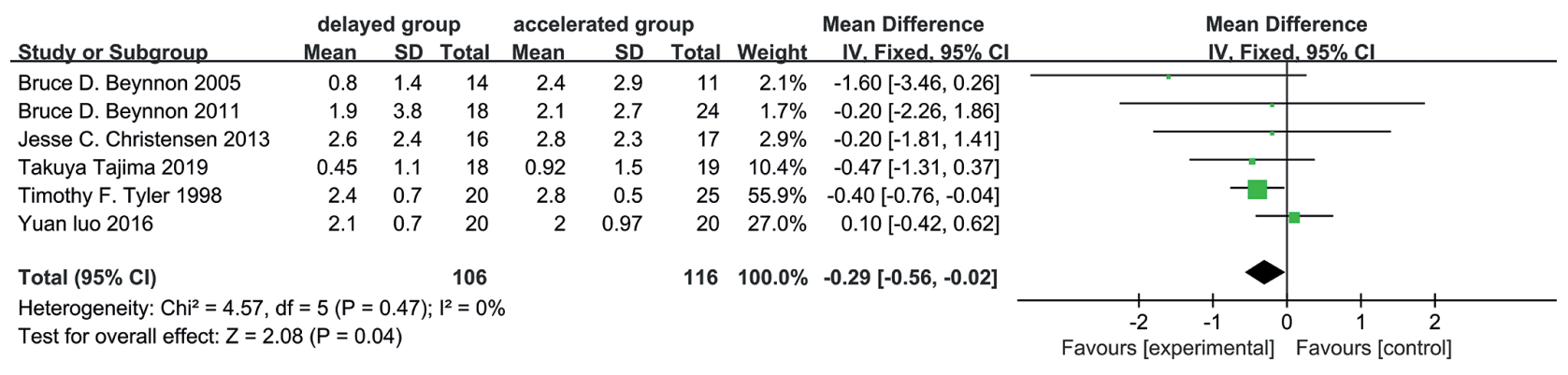

Fig. 7. Pooled analysis for A-P knee laxity (side-to-side difference). SD: standard deviation; $95 \%$ CI: $95 \%$ confidence interval; IV: inverse variance method. 


\section{DISCUSSION}

This systematic review and meta-analysis of 9 studies included randomized controlled, retrospective, and prospective trials comparing delayed with accelerated weight-bearing after ACLR.

Based on the data for 151 delayed and 171 accelerated weight-bearing patients, IKDC scores were higher in the accelerated group than in the delayed group, and Lysholm, Tegner and KOOS scores were independent of the group allocation. The accelerated weight-bearing group showed increased risk of knee laxity and bone tunnel enlargement. Most studies reported that there was no statistical difference between the two groups in quadriceps strength and ROM at 2 years follow-up, although the accelerated weight-bearing group performed better in the early stages. Only one study showed that delayed weight-bearing was detrimental to both quadriceps strength and ROM, possibly due to disuse muscle atrophy (13).

Five studies chose hamstring tendon (HT) as grafts to reconstruct ACL, while 4 studies chose bonepatellar tendon-bone (BPTB) grafts. Most orthopaedic surgeons still consider these 2 procedures to be the standard for ACL reconstruction, despite morbidities, including difficulty kneeling, patellar fracture, postoperative anterior knee pain, weakness in hip extension, terminal knee flexion, and variable graft sizes and lengths. However, these 2 grafts have a similar performance in knee laxity. No study showed any difference between the BPTB and HT groups for laxity in Freedman's review (19). Based on a metaanalysis, which included 15 studies, Samuelsen put forward the same view (20). There were differences in the time from injury to surgery in the included studies, ranging from immediate surgery to 6 months later. Of patients with acute ACL injury, 55.2\% have bone marrow oedema. Hanypsiak et al. (21) did not find a correlation between bone marrow oedema and clinical outcome at 12 year follow-up. In Christoffer's report, acute and delayed ACLR also provided comparable knee laxity after 12 months (22).

The ACL is an essential element for controlling tibial motion produced by an axial tibial force (1). Tensile forces may develop in the ACL under physiological levels of tibio-femoral (TF) joint compressive loading because of the inherent posterior tilt of the tibial plateau of $10-15^{\circ}$. There is little doubt that weightbearing training influences the weak tendon-bone interface after ACLR, especially in people with higher steep posterior tibial slopes. The effect of mechanical stimulation on the graft and bone tunnel in the early post-reconstruction period has always been the focus of debate. Song et al. (23) evaluated the effect of mecha- nical loading on tendon-bone healing in a rabbit model, and found that mechanical loading elevated ultimate load-to-failure levels. However, some reports suggest that less mechanical loading causes less scar tissue, decreased expression of matrix metalloproteinase 13 (MMP-13), and a more organized tendon-bone interface (24-26). The ACL graft ligamentization process is considered a critical histological factor in the process of rehabilitation. It involves successive stages of inflammation and necrosis, followed by revascularization, cellular repopulation, and new matrix synthesis. A more continuous tendon-bone interface can provide better healing, reduce micro-movement of the graft in the tunnel and the occurrence of knee laxity. Rehabilitation protocols that cause mechanical stimulation at the tendon-bone interface, such as weight-bearing protocols, should be planned with caution, considering the accelerated healing modulated by inflammation processes.

Accelerated weight-bearing rehabilitation programmes after an ACLR are increasingly used by orthopaedic surgeons $(2,27-33)$. However, the role of early weight-bearing is yet to be widely accepted. Biomechanical evaluations of knee joint response under torsional or compressive loading have been performed at low force levels and do not compare with weight-bearing load transmission (34). Tyler et al. (8) reported a significant decrease in patellofemoral pain, from $35 \%$ to $8 \%$, and no effect on knee extension ROM, vastus medialis oblique function or knee stability. Many reviews have adopted the conclusions of Tyler's literature when recommending weight-bearing schemes $(30,31,33,35)$. Van Melick et al. (35) proposed practice guidelines that immediate weight-bearing should be tolerated only if there is a correct gait pattern and no pain, effusion, or an increase in temperature when walking. They also concluded that immediate weight-bearing did not affect knee laxity. Wright et al. (2) reported that full weight-bearing without support was not an influential risk factor for 2-year outcomes (IKDC, all KOOS subscales, and the Marx activity rating) based on the Multicenter ACL Revision Study (MARS) Group. The Orthopedic Section of the American Physical Therapy Association formulated clinical practice guidelines, recommending that early weight-bearing can be used for patients without incurring detrimental effects on stability or function (36). Clinicians may implement early weight-bearing as tolerated (within 1 week after surgery) (36). In 1 study, $68.7 \%$ of clinicians preferred their patients to initiate full weight-bearing, $18.6 \%$ preferred their patients at $50 \%$ weight-bearing. $25 \%$ weight-bearing and toe touch weight-bearing were chosen by $7.5 \%$ and $3.6 \%$ of clinicians, respectively, and non-weight-bearing was 
chosen only $1.6 \%$ of the time (29). Although the accelerated weight-bearing protocol is more popular, there have been many reports of bone tunnel enlargement $(3,37,38)$. In our opinion, accelerated weight-bearing causes more serious knee laxity at follow-up within 2 years. The mechanism of bone tunnel enlargement following ACLR is not yet fully understood. Some potential mechanisms have been suggested to explain its aetiology, such as immune response, cell necrosis in the tunnel, non-specific inflammatory response, heat necrosis when drilling, and micro-motion at the interface. Vadala et al. (38) compared bone tunnel by computed tomography (CT) between standard and brace-free, accelerated rehabilitation. The result of bone tunnel widening of the tibia and femur in the accelerated weight-bearing group indicated that conservative rehabilitation programmes might be helpful, without adverse clinical outcomes (37). Bone tunnel enlargement does not seem to affect short-term clinical outcomes according to studies published to date, but it would be premature to conclude that it has no effect on prognosis. Hoshino et al. (39) reported that stress distribution at the bone tunnel aperture is not equal in different orientations. In Taketomi's opinion, the femoral tunnel enlarges because of the stress exerted on the wall in the orientation where the graft runs and pulls on the knee, and the wall supporting the graft moves closer to the direction of the pull, resulting in increased laxity (40). Because of studies with a short follow-up time, it is challenging to evaluate the longterm effects of bone tunnel enlargement.

This systematic review indicates that conservative rehabilitation programmes may be safe. This finding differs from the current mainstream view, which is contradictory to the results of histology. Less mechanical loading causes less scar tissue, decreased expression of MMP-13, and a more organized tendon-bone interface (24-26). Macrophages expressing the ED1 antigen accumulate in the first few days following surgery (peaking at 7 days). Early weight-bearing may stimulate the generation of transforming growth factor- $\beta$ (TGF- $\beta$ ), which is one of the most important mediators of early macrophage activity in normal wound-healing. TGF- $\beta$ causes more scar tissue formation to influence the tendon-bone interface. Simultaneously, the movement of the graft changes mechanical stress at the entrance of the tunnel, resulting in further aggravation of laxity.

The current study has several limitations. The analysis of trials showed some heterogeneity about operation method, graft choice, specific plan of weight-bearing programmes, follow-up time, injuryto-surgery time, and the influence of other rehabilitation programmes. Eight studies chose a single-bundle reconstruction, and one study chose a double-bundle reconstruction. There was significant heterogeneity in graft choice. Investigators have different views on specific weight-bearing programmes. The current study defined full weight-bearing within 2 weeks as accelerated weight-bearing, and full weight-bearing beyond 2 weeks as delayed weight-bearing. Because of these limitations, it is not possible for this study to determine the best time to start weight-bearing training and the rehabilitation process. Meanwhile, different studies describe different protocols for other rehabilitation exercises other than weight-bearing, namely, time to start of continuous passive motion (CPM) or ROM of knee joint during the rehabilitation. Furthermore, the current study could not assess the impact of knee ROM exercises.

Despite these limitations, this systematic review and meta-analysis represent up-to-date research, and it was not found that accelerated weight-bearing has an adverse clinical score although it results in worse stability of the knee joint. We believe that the reason may be due to the short follow-up period, when the loss of stability has not caused bad symptoms. We predict that accelerated weight-bearing may cause meniscus injuries, cartilage injuries, or osteoarthritis down the line. Further research with longer follow-up is needed to assess the long-term prognosis.

In conclusion, this systematic review found that, after ACLR, accelerated weight-bearing rehabilitations resulted in greater laxity and bone tunnel widening than delayed weight-bearing rehabilitation, with no significant clinical improvement in outcomes. This meta-analysis recommends delayed weight-bearing and careful selection of rehabilitation protocols following ACLR to restore knee stability.

\section{ACKNOWLEDGEMENTS}

The National Natural Science Foundation of China (grant number 81701811), the Jilin Province Science and Technology Development Plan Project (grant number 20190103087JH) and the Health Special Project of Jilin Province Finance Department (grant number 2018SCZ026), supported this work.

The authors have no conflicts of interests to declare.

\section{REFERENCES}

1. Tajima T, Yamaguchi N, Nagasawa M, Morita Y, Nakamura $Y$, Chosa E. Early weight-bearing after anterior cruciate ligament reconstruction with hamstring grafts induce femoral bone tunnel enlargement: a prospective clinical and radiographic study. BMC Musculoskelet Disord 2019; 20: 274.

2. MARS Group. Rehabilitation predictors of clinical outcome following revision ACL reconstruction in the MARS cohort. J Bone Joint Surg Am 2019; 101: 779-786.

3. Rizer M, Foremny GB, Rush A, 3rd, Singer AD, Baraga M, Kaplan LD, et al. Anterior cruciate ligament reconstruction 
tunnel size: causes of tunnel enlargement and implications for single versus two-stage revision reconstruction. Skeletal Radiol 2017; 46: 161-169.

4. Harput G, Howard JS, Mattacola C. Comparison of muscle activation levels between healthy individuals and persons who have undergone anterior cruciate ligament reconstruction during different phases of weight-bearing exercises. J Orthop Sports Phys Ther 2016; 46: 984-992.

5. Chmielewski TL, Wilk KE, Snyder-Mackler L. Changes in weight-bearing following injury or surgical reconstruction of the ACL: relationship to quadriceps strength and function. Gait Posture 2002; 16: 87-95.

6. Sritharan P, Schache AG, Culvenor AG, Perraton LG, Bryant $A L$, Crossley KM. Between-limb differences in patellofemoral joint forces during running at 12 to 24 months after unilateral anterior cruciate ligament reconstruction. Am J Sports Med 2020; 48: 1711-1719.

7. Lin PE, Sigward SM. Influence of hamstrings on knee moments during loading response of gait in individuals following ACL reconstruction. J Orthop Res 2020; 38: 378-386.

8. Tyler TF, McHugh MP, Gleim GW, Nicholas SJ. The effect of immediate weightbearing after anterior cruciate ligament reconstruction. Clin Orthop Relat Res 1998: 141-148.

9. Moher D, Liberati A, Tetzlaff J, Altman DG. Preferred reporting items for systematic reviews and meta-analyses: the PRISMA statement. J Clin Epidemiol 2009; 62: 10061012.

10. Higgins JP, Altman DG, Gotzsche PC, Juni $P$, Moher D, Oxman AD, et al. The Cochrane Collaboration's tool for assessing risk of bias in randomised trials. BMJ 2011; 343: d5928.

11. Di Miceli R, Marambio CB, Zati A, Monesi R, Benedetti MG. Do knee bracing and delayed weight bearing affect midterm functional outcome after anterior cruciate ligament reconstruction? Joints 2017; 5: 202-206.

12. Luo $Y$, Shen $W$, Jiang $Z$, Sha J. Treadmill training with partial body-weight support after anterior cruciate ligament reconstruction: a randomized controlled trial. J Phys Ther Sci 2016; 28: 3325-3329.

13. Zhu W, Wang D, Han Y, Zhang N, Zeng Y. Anterior cruciate ligament $(A C L)$ autograft reconstruction with hamstring tendons: clinical research among three rehabilitation procedures. Eur J Orthop Surg Traumatol 2013; 23: 939-943.

14. Christensen JC, Goldfine LR, West HS. The effects of early aggressive rehabilitation on outcomes after anterior cruciate ligament reconstruction using autologous hamstring tendon: a randomized clinical trial. J Sport Rehabil 2013; 22: $191-201$.

15. Beynnon BD, Johnson RJ, Naud S, Fleming BC, Abate JA, Brattbakk B, et al. Accelerated versus nonaccelerated rehabilitation after anterior cruciate ligament reconstruction: a prospective, randomized, double-blind investigation evaluating knee joint laxity using roentgen stereophotogrammetric analysis. Am J Sports Med 2011; 39: 2536-2548.

16. Beynnon BD, Uh BS, Johnson RJ, Abate JA, Nichols CE, Fleming $B C$, et al. Rehabilitation after anterior cruciate ligament reconstruction: a prospective, randomized, double-blind comparison of programs administered over 2 different time intervals. Am J Sports Med 2005; 33: 347-359.

17. Henriksson M, Rockborn P, Good L. Range of motion training in brace vs. plaster immobilization after anterior cruciate ligament reconstruction: a prospective randomized comparison with a 2-year follow-up. Scand J Med Sci Sports 2002; 12: 73-80.

18. Cristiani $R$, Mikkelsen $C$, Wange $P$, Olsson $D$, Stålman $A$, Engström B. Autograft type affects muscle strength and hop performance after ACL reconstruction. A randomised controlled trial comparing patellar tendon and hamstring tendon autografts with standard or accelerated rehabilitation. Knee Surg Sports Traumatol Arthrosc 2020; 29: 3025-3036.

19. Poehling-Monaghan KL, Salem H, Ross KE, Secrist E,
Ciccotti MC, Tjoumakaris F, et al. Long-term outcomes in anterior cruciate ligament reconstruction: a systematic review of patellar tendon versus hamstring autografts. Orthop J Sports Med 2017; 5: 2325967117709735.

20. Samuelsen BT, Webster KE, Johnson NR, Hewett TE, Krych AJ. Hamstring autograft versus patellar tendon autograft for ACL reconstruction: is there a difference in graft failure rate? A meta-analysis of 47,613 patients. Clin Orthop Relat Res 2017; 475: 2459-2468.

21. Hanypsiak BT, Spindler KP, Rothrock CR, Calabrese GJ, Richmond B, Herrenbruck TM, et al. Twelve-year follow-up on anterior cruciate ligament reconstruction: long-term outcomes of prospectively studied osseous and articular injuries. Am J Sports Med 2008; 36: 671-677.

22. von Essen C, McCallum S, Barenius B, Eriksson K. Acute reconstruction results in less sick-leave days and as such fewer indirect costs to the individual and society compared to delayed reconstruction for ACL injuries. Knee Surg Sports Traumatol Arthrosc 2020; 28: 2044-2052.

23. Song $F$, Jiang D, Wang $T$, Wang $Y$, Chen $F, X u$, et al. Mechanical loading improves tendon-bone healing in a rabbit anterior cruciate ligament reconstruction model by promoting proliferation and matrix formation of mesenchymal stem cells and tendon cells. Cell Physiol Biochem 2017; 41: 875-889.

24. Hettrich CM, Gasinu S, Beamer BS, Stasiak M, Fox A, Birmingham $P$, et al. The effect of mechanical load on tendon-to-bone healing in a rat model. Am J Sports Med 2014; 42: 1233--1241.

25. Ma R, Schär M, Chen T, Sisto M, Nguyen J, Voigt C, et al. Effect of dynamic changes in anterior cruciate ligament in situ graft force on the biological healing response of the graft-tunnel interface. Am J Sports Med 2018; 46: 915-923.

26. Camp CL, Lebaschi A, Cong GT, Album Z, Carballo C, Deng $\mathrm{XH}$, et al. Timing of postoperative mechanical loading affects healing following anterior cruciate ligament reconstruction: analysis in a murine model. J Bone Joint Surg Am 2017; 99: 1382-1391.

27. Thrush C, Porter TJ, Devitt BM. No evidence for the most appropriate postoperative rehabilitation protocol following anterior cruciate ligament reconstruction with concomitant articular cartilage lesions: a systematic review. Knee Surg Sports Traumatol Arthrosc 2018; 26: 1065-1073.

28. Janssen RP, du Mée AW, van Valkenburg J, Sala HA, Tseng CM. Anterior cruciate ligament reconstruction with 4-strand hamstring autograft and accelerated rehabilitation: a 10-year prospective study on clinical results, knee osteoarthritis and its predictors. Knee Surg Sports Traumatol Arthrosc 2013; 21: 1977-1988.

29. Budny J, Fox J, Rauh M, Fineberg M. Emerging trends in anterior cruciate ligament reconstruction. J Knee Surg 2017; 30: 63-69.

30. Kruse LM, Gray BL, Wright RW. Anterior cruciate ligament reconstruction rehabilitation in the pediatric population. Clin Sports Med 2011; 30: 817-824.

31. Wright RW, Haas AK, Anderson J, Calabrese G, Cavanaugh J, Hewett TE, et al. Anterior cruciate ligament reconstruction rehabilitation: MOON guidelines. Sports Health 2015; 7: 239-243.

32. Forrester LA, Schweppe EA, Popkin CA. Variability in rehabilitation protocols following pediatric anterior cruciate ligament $(A C L)$ reconstruction. Phys Sportsmed 2019; 47: 448-454.

33. Wright RW, Preston E, Fleming BC, Amendola A, Andrish JT, Bergfeld JA, et al. A systematic review of anterior cruciate ligament reconstruction rehabilitation: part I: continuous passive motion, early weight bearing, postoperative bracing, and home-based rehabilitation. J Knee Surg 2008; 21: 217-224.

34. Meyer EG, Haut RC. Anterior cruciate ligament injury induced by internal tibial torsion or tibiofemoral compression. J Biomech 2008; 41: 3377-3383. 
35. van Melick N, van Cingel RE, Brooijmans F, Neeter $C$, van Tienen T, Hullegie W, et al. Evidence-based clinical practice update: practice guidelines for anterior cruciate ligament rehabilitation based on a systematic review and multidisciplinary consensus. $\mathrm{Br}$ J Sports Med 2016; 50: 1506-1515.

36. Logerstedt DS, Scalzitti D, Risberg MA, Engebretsen L, Webster KE, Feller J, et al. Knee stability and movement coordination impairments: knee ligament sprain revision 2017. J Orthop Sports Phys Ther 2017; 47: A1-a47.

37. Kruse LM, Gray B, Wright RW. Rehabilitation after anterior cruciate ligament reconstruction: a systematic review. J Bone Joint Surg Am 2012; 94: 1737-1748.

38. Vadalà A, Iorio R, De Carli A, Argento G, Di Sanzo V,

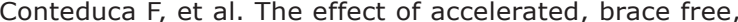
rehabilitation on bone tunnel enlargement after ACL reconstruction using hamstring tendons: a CT study. Knee Surg Sports Traumatol Arthrosc 2007; 15: 365-371.

39. Hoshino Y, Kuroda R, Nishizawa Y, Nakano N, Nagai K, Araki $D$, et al. Stress distribution is deviated around the aperture of the femoral tunnel in the anatomic anterior cruciate ligament reconstruction. Knee Surg Sports Traumatol Arthrosc 2018; 26: 1145-1151.

40. Taketomi S. Editorial commentary: tunel widening after anterior cruciate ligament reconstruction may increase laxity and complicate revision. Arthroscopy 2021; 37: 2564-2566. 